\title{
La rehabilitación vestibular es una terapia efectiva para los mareos crónicos en atención primaria
}

\section{Objetivo}

Evaluar la efectividad de ejercicios de rehabilitación vestibular, enseñada por enfermeras de atención primaria, para disminuir los síntomas en pacientes con mareo crónico vs. tratamiento habitual.

\section{Diseño}

Estudio aleatorizado cruzado (cross-over), controlado, simple ciego.

Lugar

Veinte centros de atención primaria, Inglaterra.

\section{Pacientes}

Pacientes adultos $(n=170)$ con mareo crónico de diferentes etiologías (se excluyeron aquellos con causa no laberíntica de mareo, enfermedad grave del sistema nervioso central, contraindicación para realizar movimientos de cuello, mareos de menos de dos meses de evolución en los dos años previos, y los que no presentaban síntomas al realizar los movimientos de rehabilitación vestibular.

\section{Intervención}

Se aleatorizó a los pacientes a recibir rehabilitación vestibular (RV) $(n=83)$ o tratamiento médico habitual (TM) $(n=87)$ en el período inicial de tres meses. Los tres meses subsiguientes se invirtió la intervención (el que recibía RV pasó a TM y viceversa). La intervención consistió en un taller de seis horas para entrenar a 23 enfermeras a enseñar a los pacientes los ejercicios. Estos consistían en una serie de movimientos de la cabeza que los pacientes debían realizar en sus casas, enseñados a través de una entrevista y un manual, y con seguimiento telefónico para evaluar el cumplimiento y apoyar la continuidad del tratamiento. Los pacientes podían continuar con su tratamiento habitual, iniciar nuevos o consultar a especialistas.

\section{Medición de resultados principales}

Resultados principales: mareos espontáneos y provocados luego de los ejercicios (autoreporte), calidad de vida en relación al mareo (autoreporte) y estabilidad postural (medida con un oscilómetro portátil). Resultados secundarios: ansiedad, depresión y funcionamiento físico. El análisis fue por intención de tratar.

\section{Resultados principales}

Se evidenció mejoría en todos los resultados principales en el grupo RV. El riesgo relativo para mejoría clínica significativa en la escala de vértigo fue de 1,78. También se puso de manifiesto la disminución de los síntomas provocados por la realización de los ejercicios en el grupo intervención (ver tabla).

\section{Tabla. Resultados Principales}

\begin{tabular}{l|c|c|c|c|c}
\multicolumn{1}{c|}{ Resultado } & $\begin{array}{c}\text { RV } \\
(\mathbf{n}=\mathbf{8 3})\end{array}$ & $\begin{array}{c}\text { TM } \\
(\mathbf{n}=\mathbf{8 7})\end{array}$ & $\begin{array}{c}\mathbf{R R} \\
(\text { IC } 95 \%)\end{array}$ & $\begin{array}{c}\text { NWT } \\
\text { (IC 95\%) }\end{array}$ & p \\
$\begin{array}{l}\text { Mejoría en la escala } \\
\text { de Vértigo }\end{array}$ & $67 \%$ pacientes & $38 \%$ pacientes & $\begin{array}{c}1,78 \\
(1,31-2,42)\end{array}$ & $\begin{array}{c}3,44 \\
(2 \text { a } 7)\end{array}$ & $<0.0001$ \\
\hline $\begin{array}{l}\text { Disminución de } \\
\text { síntomas provocados }\end{array}$ & $23 \%$ pacientes & $6 \%$ pacientes & $\begin{array}{c}4,03 \\
(1,58-10,30)\end{array}$ & $\begin{array}{c}5,88 \\
(4 \text { a 13) }\end{array}$ & 0.002 \\
\hline
\end{tabular}

A los seis meses, el grupo RV mantuvo la mejoría obtenida a los tres meses; $y$ no hubo diferencia significativa entre ambos grupos a los seis meses (luego de que se aplicara la RV en el grupo que comenzaba con TM). No hubo diferencias significativas en los resultados secundarios. La adherencia a los ejercicios fue buena y con buena calidad de los reportes, y no se informaron efectos adversos graves.

\section{Conclusiones}

La rehabilitación vestibular realizada y supervisada por enfermeras de atención primaria, reduce los síntomas, la discapacidad y alteraciones resultantes del mareo crónico; y esta mejoría se mantiene al menos tres meses.

Fuente de financiamiento: Directorate of Health and Social Care South, London, United Kingdom.

\section{Comentario}

Los mareos, vértigo y alteraciones del equilibrio son síntomas muy comunes que se presentan en un 5 a $10 \%$ de los pacientes que consultan en atención primaria, y son una causa importante de las consultas a especialistas (neurólogos, otorrinolaringólogos). Son mas frecuentes en pacientes mayores de 75 años.

La rehabilitación vestibular consiste en una serie de ejercicios graduados que tienen como objetivo estimular el sistema vestibular (alterado en los pacientes con mareos) para promover la adaptación neurológica. ${ }^{1-2}$ Ayudan a vencer el miedo, disminuyen la evitación de actividades y promueven la recuperación de la confianza en el equilibrio. Estos ejercicios (seis en total) consisten en mover la cabeza de lado a lado, arriba y abajo, y estos combinados con ojos abiertos y cerrados, y enfocando un objeto fijo. Al principio se realizan lentamente estando sentados y luego se realizan parados o caminando, aumentando así la dificultad.

Este estudio demuestra que los ejercicios de rehabilitación vestibular realizados diariamente reducen los síntomas, la inestabilidad postural, la restricción en la vida diaria, y las caídas provocadas por el mareo.
Provee clara evidencia de los beneficios de una intervención breve llevada a cabo por enfermeras de atención primaria que recibieron previamente un mínimo entrenamiento.

Es una herramienta simple, costo-efectiva, sin efectos adversos, fácilmente aplicable en el ámbito de la atención primaria, y que ayudaría a disminuir el número de drogas prescriptas y las consultas a los especialistas.

Por lo tanto, en el manejo de pacientes con vértigo y mareo deberíamos tratar las causas "tratables", y motivar a nuestros pacientes a realizar ejercicio físico y reentrenamiento del equilibrio para no abandonar sus actividades físicas y sociales, o tener que acudir a andadores o bastones.

\section{Conclusiones del comentador}

Este estudio nos otorga a los médicos de atención primaria una opción de tratamiento efectiva para los pacientes con mareos crónicos.

\section{Dr Tomás Smith [ Unidad de Medicina Familiar y Preventiva. Hospital Italiano de Buenos Aires. ]}

Smith T. La rehabilitación vestibular es una terapia efectiva para los mareos crónicos en atención primaria. Evid. actual. práct. ambul. 2005;8:72. Comentado de: Yardley L, Donovan-Hall M. et al. Effectiveness of primary care-based vestibular rehabilitation for chronic dizziness. Ann Intern Med. 2004;141: 598-605. PMID: 15492339

\section{Referencias}

1. Yardley L, Beech S, Zander L. A randomized controlled trial of exercise therapy for dizziness and vertigo in primary care. Br J Gen Pract. 1998;48:1136-40.

2. Dieterich M. Easy, Inexpensive, and Effective: Vestibular Exercises for Balance Control. Editorial. Annals Intern Med. 2004 Oct. Vol 141: № 8 